

\title{
Highway Vehicular Delay Tolerant Networks: Information Propagation Speed Properties
}

Emmanuel Baccelli, Philippe Jacquet, Bernard Mans, Georgios Rodolakis

\section{To cite this version:}

Emmanuel Baccelli, Philippe Jacquet, Bernard Mans, Georgios Rodolakis. Highway Vehicular Delay Tolerant Networks: Information Propagation Speed Properties. IEEE Transactions on Information Theory, 2012, 58 (3), pp.1743 - 1756. 10.1109/TIT.2012.2187734 . hal-00651597

\section{HAL Id: hal-00651597 https://hal.science/hal-00651597}

Submitted on 13 Dec 2011

HAL is a multi-disciplinary open access archive for the deposit and dissemination of scientific research documents, whether they are published or not. The documents may come from teaching and research institutions in France or abroad, or from public or private research centers.
L'archive ouverte pluridisciplinaire HAL, est destinée au dépôt et à la diffusion de documents scientifiques de niveau recherche, publiés ou non, émanant des établissements d'enseignement et de recherche français ou étrangers, des laboratoires publics ou privés. 


\title{
Highway Vehicular Delay Tolerant Networks: Information Propagation Speed Properties
}

\author{
Emmanuel Baccelli*, Philippe Jacquet*, Bernard Mans ${ }^{\dagger}$, Georgios Rodolakis ${ }^{\dagger}$ \\ *INRIA, France, \{firstname.lastname $\}$ @inria.fr \\ ${ }^{\dagger}$ Macquarie University, Australia, \{firstname.lastname\}@mq.edu.au
}

\begin{abstract}
In this paper, we provide a full analysis of the information propagation speed in bidirectional vehicular delay tolerant networks such as roads or highways. The provided analysis shows that a phase transition occurs concerning the information propagation speed, with respect to the vehicle densities in each direction of the highway. We prove that under a certain threshold, information propagates on average at vehicle speed, while above this threshold, information propagates dramatically faster at a speed that increases quasi-exponentially when the vehicle density increases. We provide the exact expressions of the threshold and of the average information propagation speed near the threshold, in case of finite or infinite radio propagation speed. Furthermore, we investigate in detail the way information propagates under the threshold, and we prove that delay tolerant routing using cars moving on both directions provides a gain in propagation distance, which is bounded by a sub-linear power law with respect to the elapsed time, in the referential of the moving cars. Combining these results, we thus obtain a complete picture of the way information propagates in vehicular networks on roads and highways, which may help designing and evaluating appropriate VANET routing protocols. We confirm our analytical results using simulations carried out in several environments (The One and Maple).
\end{abstract}

\section{INTRODUCTION}

The limits of the performance of multi-hop packet radio networks have been studied for more than a decade, yielding fundamental results such as those of Gupta and Kumar [12] on the capacity of fixed ad hoc networks. These studies assume that either end-to-end paths are available or packets are dropped on the spot. Following seminal works such as [11] evaluating the potential of mobility to increase capacity, recent research studies focussed on the limits of the performance beyond the end-to-end hypothesis, i.e., when end-to-end paths may not exist and communication routes may only be available through time and mobility. In this context nodes may carry packets for a while until advancing further towards the destination is possible. Such networks are generally referred as Intermittently Connected Networks (ICNs) or Delay Tolerant Networks (DTNs). Interest in DTN modeling and analysis has risen as novel network protocols and architectures are being elaborated to accommodate various forms of new, intermittently connected networks, which include vehicular ad hoc networks (VANETs), power-saving sensor networks, or even Interplanetary Internet [7].

In this paper, we study the information propagation speed in the typical case of bidirectional vehicular DTNs, such as roads or highways (e.g., about $75 \%$ of the total statute miles in the USA [18]). Our objective consists in determining the maximum speed at which a packet (or beacon) of information can propagate in such a bidirectional vehicular network. Our analysis shows that a phase transition occurs concerning information propagation speed, with respect to the vehicle density. We prove that under a certain threshold, information propagates on average at vehicle speed, while above this threshold, information propagates much faster. We provide the exact expressions of the threshold and of the average propagation speed near the threshold.

With applications such as safety, ad hoc vehicular networks are receiving increasing attention (see recent surveys [6], [17]). Delay tolerant architectures have thus been considered in this context in recent studies, and various analytical models have been proposed. In [19], the authors study vehicle traces and conclude that vehicles are very close to being exponentially distributed on highways. The authors of [5] also base themselves on traces gathered in DieselNET (the experimental vehicular network deployed by UMass) to elaborate and evaluate a novel DTN routing algorithm. In [10], the authors provide a model for critical message dissemination in vehicular networks and derive results on the average delay in delivery of messages with respect to vehicle density. The authors of [21] propose an alternative model for vehicular DTNs and derived results on node connectivity, under the hypothesis that vehicles are exponentially distributed. The study is based on queuing theory techniques and characterizes the relationship between node connectivity and several parameters including speed distribution and traffic flow. In [20], the authors model vehicles on a highway, and study message propagation among vehicles in the same direction, taking into account speed differences between vehicles, while in [16] authors study message dissemination among vehicles in opposing directions and conclude that using both directions increases dissemination significantly.

Several studies focus on characterizing the packet propagation delay in DTNs: [8] which models DTNs as ErdösRényi random graphs to derive results concerning packet propagation delay, [22] which uses fluid limit techniques to derive relationships between buffer space, packet duplication and dissemination delay. Other studies focus on information propagation speed in DTNs. In [15] the authors show that when a two-dimensional network is not percolated, the latency scales linearly with the Euclidean distance between the sender and the receiver, while in [13], the authors obtained analytical estimates of the constant bounds on the speed at which information can propagate in two-dimensional DTNs. Studies 
such as [1], [2], [3] are the closest related work, also focusing on information propagation speed in one-dimensional DTNs. These studies introduce a model based on space discretization to derive upper and lower bounds in the highway model under the assumption that the radio propagation speed is finite. Their bounds, although not converging, clearly indicates the existence of a phase transition phenomenon for the information propagation speed. Comparatively, we introduce a model based on Poisson point process on continuous space, that allows both infinite and finite radio propagation speed, and derive more fine-grained results above and below the threshold (some of the work described in the following was presented in [4]). Using our model, we prove and explicitly characterize the phase transition.

In this context, our contributions are as follows: (1) we develop a new vehicule-to-vehicule model for information propagation in bidirectional vehicular DTNs in Section II; (2) we show the existence of a threshold (with respect to the vehicle density), above which the information speed increases dramatically over the vehicle speed, and below which the information propagation speed is on average equal to the vehicle speed, and (3) we give the exact expression of this threshold, in Section III; (4) in Section IV, we prove that, under the threshold, even though the average propagation speed equals the vehicle speed, DTN routing using cars moving on both directions provides a gain in the propagation distance, and this gain follows a sub-linear power law with respect to the elapsed time, in the referential of the moving cars; (5) we characterize information propagation speed as increasing quasi-exponentially with the vehicle density when the latter becomes large above the threshold, in Section V; (6) we cover both infinite radio propagation speed cases, then finite radio propagation speed cases in Section VI, and (7) we validate the provided analysis with simulations in multiple environments (The One and Maple), in Section VII.

\section{Model And Results}

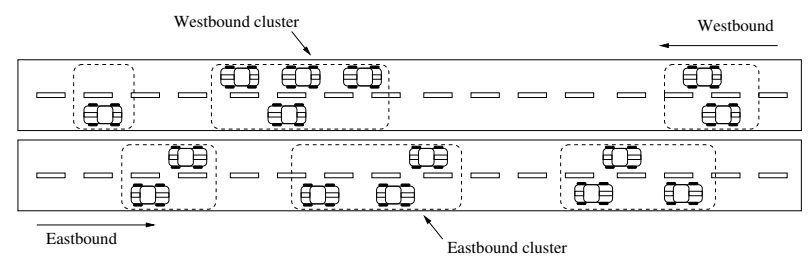

Fig. 1. Model of a bidirectional vehicular network on a highway.

In the following, we consider a bidirectional vehicular network, such as a road or a highway, where vehicles move in two opposite directions (say east and west, respectively) at speed $v$, as depicted in Figure 1. Let us consider eastbound vehicle density as Poisson with intensity $\lambda_{e}$, while westbound vehicle density is Poisson with intensity $\lambda_{w}$. We note that the Poisson distribution is indeed a reasonable approximation of vehicles moving on non-congested highways [19]. Furthermore, we consider that the radio propagation speed (including store and

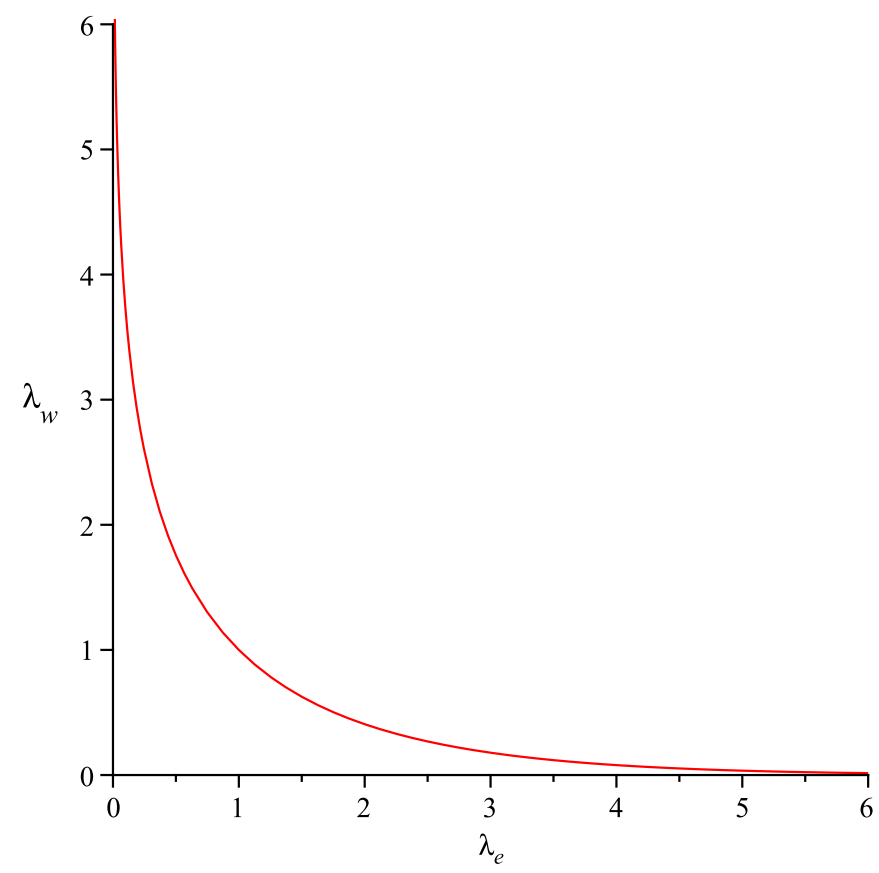

Fig. 2. Information propagation threshold with respect to $\left(\lambda_{e}, \lambda_{w}\right)$. Below the curve, the average information propagation speed is limited to the vehicle speed (i.e., the propagation speed is 0 in the referential of the eastbound cars), while above the curve, information propagates faster on average.

forward processing time) is infinite, and that the radio range of each transmission in each direction is of length $R$.

The main result presented in this paper is that, concerning the information propagation speed in such an environment, a phase transition occurs when $\lambda_{e} R$ and $\lambda_{w} R$ coincide on the curve $y=x e^{-x}$, i.e.,

$$
\lambda_{e} R e^{-\lambda_{e} R}=\lambda_{w} R e^{-\lambda_{w} R} .
$$

Figure 2 shows the corresponding threshold curve for $R=1$ (in the following, we will always consider the case $R=1$, without loss of generality). We show that below this threshold, the average information propagation speed is limited to the vehicle speed, while above the curve, information propagates faster on average.

We focus on the propagation of information in the eastbound lane. Our aim is the evaluation of the maximum speed at which a packet (or beacon) of information can propagate. An information beacon propagates in the following manner, illustrated in Figure 3: it moves toward the east jumping from car to car until it stops because the next car is beyond radio range. The propagation is instantaneous, since we assume that radio routing speed is infinite. The beacon waits on the last eastbound car until the gap is filled by westbound cars, so that the beacon can move again to the next eastbound car.

We denote $\mathbf{T}_{i}$ the duration the beacon waits when blocked for the $i$ th time and $\mathbf{D}_{i}$ the distance traveled by the beacon just after. The random variables $\mathbf{T}_{i}$ and $\mathbf{D}_{i}$ are dependent but, due to the Poisson nature of vehicle traffic, the tuples in the 


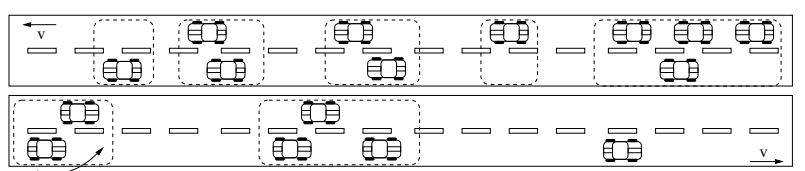

(a)



(b)

Fig. 3. Eastbound information propagation: the beacon waits on the last eastbound car (a), until the gap is bridged by westbound cars so that the beacon can move again (b).

sequence $\left(\mathbf{T}_{i}, \mathbf{D}_{i}\right)$ are i.i.d.. From now on, we denote $(\mathbf{T}, \mathbf{D})$ the independent random variable.

We denote $L(t)$ the distance traveled by the beacon during a time $t$ on the eastbound lane. We consider the distance traveled with respect to the referential of the eastbound cars. We also define the average information propagation speed $v_{p}$ as:

$$
v_{p}=\lim _{t \rightarrow \infty} \frac{\mathbf{E}(L(t))}{t} .
$$

By virtue of the renewal processes, we have

$$
v_{p}=\frac{\mathbf{E}(\mathbf{D})}{\mathbf{E}(\mathbf{T})} .
$$

We prove that $v_{p}$ is well-defined because $\mathbf{E}(\mathbf{D})$ is finite, in Section III-F. For the remainder of the paper, for $x>0$, we denote $x^{*}$ the conjugate of $x$ with respect to the function $x e^{-x}$ : $x^{*}$ is the alternate solution of the equation $x^{*} e^{-x^{*}}=x e^{-x}$. Notice that $x^{* *}=x$ and $1^{*}=1$.

We prove the following theorems, concerning the information propagation phase transition threshold (Theorem 1), the distribution of the waiting time spent by information packets in the phase depicted in Figure 3a (Theorem 2), and the total propagation distance achieved (under the phase transition threshold) because of multi-hop bridging using nodes in both traffic directions, as depicted in Figure $3 b$ (Theorem 3).

Theorem 1. For all $\left(\lambda_{e}, \lambda_{w}\right)$, the information propagation speed $v_{p}$ with respect to the referential of the eastbound cars is $v_{p}<\infty$, and,

$$
\begin{aligned}
& \lambda_{e}<\lambda_{w}^{*} \Rightarrow v_{p}=0, \\
& \lambda_{e}>\lambda_{w}^{*} \Rightarrow v_{p}>0 .
\end{aligned}
$$

Theorem 2. When $t \rightarrow \infty$,

$$
P(\mathbf{T}>t)=A\left(\lambda_{e}, \lambda_{w}\right)(2 v t)^{-\frac{\lambda_{e}}{\lambda_{w}^{*}}}(1+o(1)),
$$

for some $A\left(\lambda_{e}, \lambda_{w}\right)$, function of $\left(\lambda_{e}, \lambda_{w}\right)$.

Notice that Theorem 1 is in fact a corollary of Theorem 2.
Theorem 3. When $\lambda_{w}^{*}>\lambda_{e}\left(\right.$ case $\left.v_{p}=0\right)$, when $t \rightarrow \infty$,

$$
\mathbf{E}(L(t))=B\left(\lambda_{e}, \lambda_{w}\right)(2 v t)^{\frac{\lambda_{e}}{\lambda_{w}^{e}}}+O\left(t^{\frac{\lambda_{e}}{\lambda_{w}^{w}}}-1\right) .
$$

for some $B\left(\lambda_{e}, \lambda_{w}\right)$, function of $\left(\lambda_{e}, \lambda_{w}\right)$.

\section{Phase Transition: Proof of Theorem 1}

\section{A. Proof Outline}

We call cluster a maximal sequence of cars such that two consecutive cars are within radio range. A westbound (respectively, eastbound) cluster is a cluster made exclusively of westbound (respectively, eastbound) cars. A full cluster is made of westbound and eastbound cars.

We define the length of the cluster as the distance between the first and last cars augmented by a radio range. We denote $L_{w}$ a westbound cluster length. We start by proving in Section III-B that the Laplace transform of $L_{w}$ : $f_{w}(\theta)=\mathbf{E}\left(e^{-\theta L_{w}}\right)$ equals:

$$
f_{w}(\theta)=\frac{\left(\lambda_{w}+\theta\right) e^{-\lambda_{w}-\theta}}{\theta+\lambda_{w} e^{-\lambda_{w}-\theta}},
$$

and the exponential tail of the distribution of $L_{w}$ is given by

$$
P\left(L_{w}>x\right)=\Theta\left(e^{-\lambda_{w}^{*} x}\right)
$$

To evaluate how information will propagate according to Figure 3, we compute the distribution of the gap length $G_{e}$ between the cluster of eastbound cars on which the beacon is blocked and the next cluster of eastbound cars. We show in Section III-D that $P\left(G_{e}>x\right)=O\left(e^{-\lambda_{e} x}\right)$.

Now, let $\mathbf{T}(x)$ be the time needed to meet a westbound cluster long enough to fill a gap of length $x$ (i.e., a westbound cluster of length larger than $x$ ). We show in Section III-C that:

$$
\mathbf{E}(\mathbf{T}(x))=\Theta\left(\frac{1}{v P\left(L_{w}>x\right)}\right)=\Theta\left(e^{\lambda_{w}^{*} x}\right) .
$$

Therefore, the average time $\mathbf{T}$ to get a bridge over all possible gaps is

$$
\begin{aligned}
\mathbf{E}(\mathbf{T}) & =\int_{1}^{\infty} \mathbf{E}(\mathbf{T}(x)) e^{-x \lambda_{e}} d x \\
& =\frac{1}{2 v} \int_{1}^{\infty} \Theta\left(\exp \left(\left(\lambda_{w}^{*}-\lambda_{e}\right) x\right)\right) d x .
\end{aligned}
$$

As a result, the threshold with respect to $\left(\lambda_{w}, \lambda_{e}\right)$ where $\mathbf{E}(\mathbf{T})$ diverges is clearly when we have:

$$
\lambda_{w}^{*}=\lambda_{e},
$$

or, in other words, since $\lambda_{w}^{*} e^{-\lambda_{w}^{*}}=\lambda_{w} e^{-\lambda_{w}}$, when we have:

$$
\lambda_{w} e^{-\lambda_{w}}=\lambda_{e} e^{-\lambda_{e}} .
$$




\section{B. Cluster Length Distribution}

Lemma 1. The Laplace transform of the westbound cluster length $f_{w}(\theta)=\mathbf{E}\left(e^{-\theta L_{w}}\right)$ satisfies:

$$
f_{w}(\theta)=\frac{\left(\lambda_{w}+\theta\right) e^{-\lambda_{w}-\theta}}{\theta+\lambda_{w} e^{-\lambda_{w}-\theta}} .
$$

Proof: The length of the cluster is counted from the first car. The random variable $L_{w}$ satisfies:

- $L_{w}=1$, with probability $e^{-\lambda_{w}}$, when the first car has no car behind within the radio range;

- $L_{w}=g_{w}+L_{w}$, with probability $1-e^{-\lambda_{w}}$, where $g_{w}$ is the distance to the next car and $g_{w}<1$.

Translating this in terms of Laplace transforms yields:

$$
\begin{aligned}
f_{w}(\theta) & =e^{-\lambda_{w}-\theta}+f_{w}(\theta) \int_{0}^{1} \lambda_{w} e^{-\left(\lambda_{w}+\theta\right) x} d x \\
& =e^{-\lambda_{w}-\theta}+f_{w}(\theta) \frac{\lambda_{w}}{\lambda_{w}+\theta}\left(1-e^{-\lambda_{w}-\theta}\right) .
\end{aligned}
$$

In passing, we get $\mathbf{E}\left(L_{w}\right)=-f_{w}^{\prime}(0)=\frac{e^{\lambda} w-1}{\lambda_{w}}$.

Lemma 2. We have the asymptotic formula:

$$
P\left(L_{w}>x\right)=\frac{\left(\lambda_{w}-\lambda_{w}^{*}\right) e^{\lambda_{w}^{*}-\lambda_{w}}}{\left(1-\lambda_{w}^{*}\right) \lambda_{w}^{*}} e^{-\lambda_{w}^{*} x}(1+o(1))
$$

Proof: The asymptotics on $P\left(L_{w}>x\right)$ are given by inverse Laplace transform:

$$
\begin{aligned}
P\left(L_{w}>x\right) & =-\frac{1}{2 i \pi} \int_{-\varepsilon-i \infty}^{-\varepsilon+i \infty} \frac{f_{w}(\theta)}{\theta} e^{\theta x} d \theta \\
& =-\frac{1}{2 i \pi} \int_{-\varepsilon-i \infty}^{-\varepsilon+i \infty} \frac{\left(\lambda_{w}+\theta\right) e^{-\lambda_{w}}}{\left(\theta e^{\theta}+\lambda_{w} e^{-\lambda_{w}}\right) \theta} e^{\theta x} d \theta,
\end{aligned}
$$

for some $\varepsilon>0$ small enough. For $\Re(\theta)<0$ the denominator $\left(\theta e^{\theta}+\lambda_{w} e^{-\lambda_{w}}\right)$ has two simple roots at $\theta=-\lambda_{w}^{*}$ and $\theta=-\lambda_{w}$ and is absolutely integrable elsewhere. The root $-\lambda_{w}$ does not lead to a singularity since it is canceled by the numerator $\lambda_{w}+\theta$. The residues theorem neutralizes the pole at $-\lambda_{w}$, therefore for some $\varepsilon>0$ :

$$
P\left(L_{w}>x\right)=\frac{\left(\lambda_{w}-\lambda_{w}^{*}\right) e^{\lambda_{w}^{*}-\lambda_{w}}}{\left(1-\lambda_{w}^{*}\right) \lambda_{w}^{*}} e^{-\lambda_{w}^{*} x}+O\left(e^{-\left(\lambda_{w}^{*}+\varepsilon\right) x}\right) .
$$

\section{Road Length to Bridge a Gap}

Now, let us assume that we want to fill a gap of length $x$. We want to know the average length of westbound road until the first cluster that has a length greater than $x-1$. Figure 4 depicts a gap of length $x$, and the length of westbound road until a cluster is encountered which can bridge the gap. Let $f_{w}(\theta, x)$ be the Laplace transform of the cluster length, under the condition that it is smaller than $x: f_{w}(\theta, x)=\mathbf{E}\left(1_{\left(L_{w}<x\right)} e^{-\theta L_{w}}\right)$.

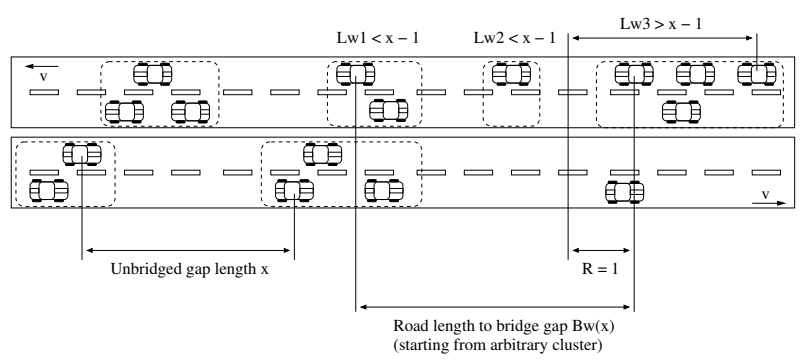

(a)

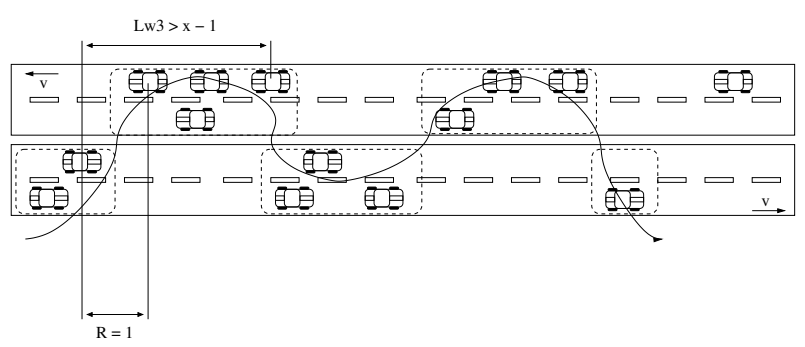

(b)

Fig. 4. Illustration of the road length $B_{w}(x)$ until a gap $x$ is bridged: (a) smaller clusters cannot bridge the gap, (b) until a westbound cluster of length at least $x-1$ is encountered.

Lemma 3. The Laplace transform of the road length $B_{w}(x)$ to bridge a gap of length $x$, starting from the beginning of an arbitrary cluster, is:

$$
\beta_{w}(\theta, x)=\mathbf{E}\left(e^{-\theta B_{w}(x)}\right)=\frac{P\left(L_{w}>x-1\right)}{1-\frac{\lambda_{w}}{\lambda_{w}+\theta} f_{w}(\theta, x-1)},
$$

and,

$\mathbf{E}\left(B_{w}(x)\right)=\left(1+O\left(e^{-\varepsilon x}\right)\right) \frac{e^{\lambda_{w}}}{\lambda_{w}} \frac{\left(1-\lambda_{w}^{*}\right) \lambda_{w}^{*}}{\left(\lambda_{w}-\lambda_{w}^{*}\right) e^{\lambda_{w}^{*}-\lambda_{w}}} e^{(x-1) \lambda_{w}^{*}}$.

Proof: Before a cluster of length greater than $x$ appears there is a succession of clusters $L_{1}, L_{2}, \ldots$ each of length smaller than $x$ (see Figure $4 \mathrm{~b}$ ). Several cases are possible:

- the first cluster to come is a cluster of length greater than $x$, with probability $P\left(L_{w}>x-1\right)$; the road length to this cluster is equal to 0 .

- the first cluster greater than $x$ is the second cluster; in this case, the road length Laplace transform is equal to $f_{w}(\theta, x) \frac{\lambda_{w}}{\lambda_{w}+\theta}$, i.e., the Laplace transform of a cluster multiplied the Laplace transform of the exponentially distributed inter-cluster distance, namely $\frac{\lambda_{w}}{\lambda_{w}+\theta}$ (as Laplace transform multiplication represents the addition of the corresponding independent random variables).

- or, in general, the first cluster greater than $x$ is the $k$ th cluster; in this case, the road length Laplace transform is equal to $\left(f_{w}(\theta, x) \frac{\lambda_{w}}{\lambda_{w}+\theta}\right)^{k}$

Therefore, the Laplace transform of the road length to the cluster of length greater than $x$ is equal to the sum of the Laplace transforms of the previous cases, i.e., $P\left(L_{w}>x-\right.$ 1) $\sum_{k=0}^{\infty}\left(f_{w}(\theta, x) \frac{\lambda_{w}}{\lambda_{w}+\theta}\right)^{k}$. 
Thus, the average is (combining with Lemma 2):

$$
\begin{aligned}
\mathbf{E}\left(B_{w}(x)\right)= & -\frac{\partial}{\partial \theta} \beta_{w}(0, x) \\
= & -\left(\frac{\partial}{\partial \theta} f_{w}(0, x-1)-\frac{1}{\lambda_{w}} f_{w}(0, x-1)\right) \\
& \times \frac{1}{P\left(L_{w}>x-1\right)} \\
= & \left(\frac{e^{\lambda_{w}}}{\lambda_{w}}+O\left(e^{-(x-1) \lambda_{w}^{*}}\right)\right) \frac{1}{P\left(L_{w}>x-1\right)} \\
= & \left(1+O\left(e^{-\varepsilon x}\right)\right) \frac{e^{\lambda_{w}}\left(1-\lambda_{w}^{*}\right) \lambda_{w}^{*}}{\lambda_{w}\left(\lambda_{w}-\lambda_{w}^{*}\right) e^{\lambda_{w}^{*}-\lambda_{w}}} e^{(x-1) \lambda_{w}^{*}}
\end{aligned}
$$

The Laplace transform of the westbound cluster length without eastbound cars, defined for all $\Re(\theta)>\lambda_{w}^{*}+\lambda_{e}$, equals:

$$
\mathbf{E}\left(e^{-\theta L_{w}^{*}}\right)=\frac{\int_{0}^{\infty} p_{w}(x) e^{-\lambda_{e} x} e^{-\theta x} d x}{\operatorname{Pr}(\text { no eastbound })}=\frac{f_{w}\left(\theta+\lambda_{e}\right)}{f_{w}\left(\lambda_{e}\right)} .
$$

and the Laplace transform of $G_{e}=L_{w}^{*}+I_{e}$ follows, as well as its average estimate.

Lemma 5. The probability density $p_{e}(x)$ of $G_{e}$ is:

$$
p_{e}(x)=\frac{\lambda_{e}}{f_{w}\left(\lambda_{e}\right)} e^{-\lambda_{e} x}\left(1+O\left(e^{-\varepsilon x}\right)\right) .
$$

Proof: The proof comes from a straightforward singularity analysis on the inverse Laplace transform.

\section{E. Distribution of Waiting Time $\mathbf{T}$}

\section{Gap Distribution}

Let us call $G_{e}$ an eastbound gap which is not bridged (see Figure 5). As illustrated in Figure 6, $G_{e}$ can be decomposed into a westbound cluster length $L_{w}^{*}$ without eastbound cars, plus a random exponentially distributed distance $I_{e}$ to the next eastbound car.

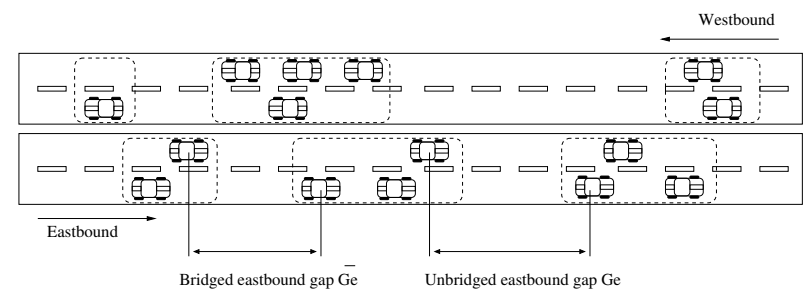

Fig. 5. Illustration of a bridged gap $\bar{G}_{e}$, and an unbridged gap $G_{e}$.



Fig. 6. Unbridged gap $G_{e}$ model; $L_{w}^{*}$ corresponds to a westbound cluster length without eastbound cars.

Lemma 4. The distribution of $G_{e}$ satisfies

$$
\mathbf{E}\left(e^{-\theta G_{e}}\right)=\frac{f_{w}\left(\theta+\lambda_{e}\right)}{f_{w}\left(\lambda_{e}\right)} \frac{\lambda_{e}}{\lambda_{e}+\theta},
$$

which is defined for all $\Re(\theta)>-\lambda_{e}$, and

$$
\mathbf{E}\left(G_{e}\right)=-\frac{f_{w}^{\prime}\left(\lambda_{e}\right)}{f_{w}\left(\lambda_{e}\right)}+\frac{1}{\lambda_{e}} .
$$

Proof: Let $p_{w}(x)$ be the probability density of a westbound cluster length $L_{w}$. The probability that a westbound cluster has no eastbound cars is:

$$
\int_{0}^{\infty} p_{w}(x) e^{-\lambda_{e} x} d x=f_{w}\left(\lambda_{e}\right)
$$

Lemma 6. We have $2 v \mathbf{T}=L_{w}^{*}+I_{w}+B_{w}-1$ (where $I_{w}$ is a random exponentially distributed distance to the next westbound car), and, therefore,

$$
2 v \mathbf{E}(\mathbf{T})=\mathbf{E}\left(L_{w}^{*}\right)-1+\frac{1}{\lambda_{w}}+\int_{1}^{\infty} \mathbf{E}\left(B_{w}(x)\right) p_{e}(x) d x
$$

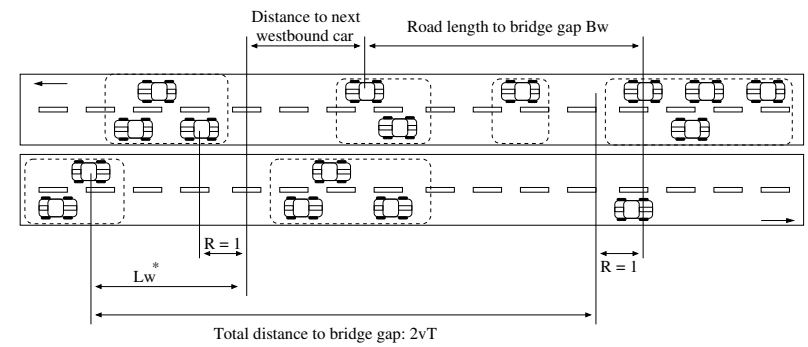

Fig. 7. Waiting time $\mathbf{T}$ : the total distance to bridge a gap is $L_{w}^{*}+I_{w}+B_{w}-1$.

Proof: The total distance to bridge a gap, as depicted in Figure 7, equals the distance to the beginning of the first westbound cluster $\left(L_{w}^{*}+I_{w}\right)$ plus the road length to bridge a gap starting from an arbitrary cluster $\left(B_{w}\right)$ minus 1 , since communication can start at exactly one radio range. Since the distance is covered by cars moving in opposite directions, we have $2 v T=L_{w}^{*}+I_{w}+B_{w}-1$. We complete the proof by taking the expectations, and averaging on all possible gap lengths $x$.

Corollary 1. The quantity $\mathbf{E}(\mathbf{T})$ converges when $\lambda_{e}>\lambda_{w}^{*}$ and diverges when $\lambda_{e}<\lambda_{w}^{*}$.

Proof: The proof comes from the leading terms of $\mathbf{E}\left(B_{w}(x)\right)$ and $p_{e}(x)$.

\section{F. Distance D Traveled after Waiting Time $\mathbf{T}$}

We denote $C_{e}$ the distance traveled beyond the first gap. As depicted in Figure 8, we have $\mathbf{D}=G_{e}+C_{e}$.

Lemma 7. The Laplace transform $\mathbf{E}\left(e^{-\theta C_{e}}\right)$ is defined for all $\Re(\theta)>-\left(\lambda_{e}+\lambda_{w}\right)^{*}$.

Proof: The random variable $C_{e}$ is smaller in probability than a full cluster. 


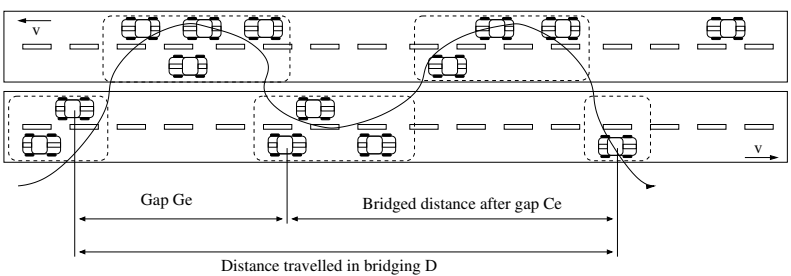

Fig. 8. Total distance $\mathbf{D}$ traveled when a bridge is created $\mathbf{D}=G_{e}+C_{e}$.

Lemma 8. The average value of $C_{e}$ satisfies:

$$
\mathbf{E}\left(C_{e}\right)=\frac{1}{\lambda_{e}} \frac{1-f_{w}\left(\lambda_{e}\right)}{f_{w}\left(\lambda_{e}\right)}+\frac{f_{w}^{\prime}\left(\lambda_{e}\right)}{f_{w}\left(\lambda_{e}\right)} .
$$

Proof: From (22), the probability that an eastbound car is not bridged to the next eastbound car equals $f_{w}\left(\lambda_{e}\right)$. The unconditional gap length is $\frac{1}{\lambda_{e}}$. We define $\bar{G}_{e}$ as an eastbound gap, under the condition that the gap is bridged (see Figure 5). Therefore, the average gap length $\bar{G}_{e}$ satisfies the following:

$$
f_{w}\left(\lambda_{e}\right) \mathbf{E}\left(G_{e}\right)+\left(1-f_{w}\left(\lambda_{e}\right)\right) \mathbf{E}\left(\bar{G}_{e}\right)=\frac{1}{\lambda_{e}},
$$

which gives $\mathbf{E}\left(\bar{G}_{e}\right)=\frac{1}{\lambda_{e}}+\frac{f_{w}^{\prime}\left(\lambda_{e}\right)}{1-f_{w}\left(\lambda_{e}\right)}$.

The distance $C_{e}$ traveled in bridging (beyond the first gap, and extended to the next cluster, which is eventually bridged) satisfies:

$$
\begin{aligned}
\mathbf{E}\left(C_{e}\right) & =\left(1-f_{w}\left(\lambda_{e}\right)\right)\left(\mathbf{E}\left(\bar{G}_{e}\right)+\mathbf{E}\left(C_{e}\right)\right) \\
& =\frac{1}{\lambda_{e}} \frac{1-f_{w}\left(\lambda_{e}\right)}{f_{w}\left(\lambda_{e}\right)}+\frac{f_{w}^{\prime}\left(\lambda_{e}\right)}{f_{w}\left(\lambda_{e}\right)} .
\end{aligned}
$$

Corollary 2. The total distance $D_{e}$ traveled including the first gap satisfies $\mathbf{E}\left(D_{e}\right)=\mathbf{E}\left(G_{e}\right)+\mathbf{E}\left(C_{e}\right)=\frac{1}{\lambda_{e} f_{w}\left(\lambda_{e}\right)}$, which remains finite for all vehicle densities.

Since $\mathbf{E}\left(D_{e}\right)$ is finite (Corollary 2) and $\mathbf{E}(\mathbf{T})$ converges when $\lambda_{e}>\lambda_{w}^{*}$, and diverges when $\lambda_{e}<\lambda_{w}^{*}$ (Corollary 1), we obtain the proof of Theorem 1 .

\section{Power Laws: Proof of Theorems 2 AND 3}

\section{A. Waiting Time Distribution}

In this section, we are interested in finding an evaluation of the waiting time distribution $P(\mathbf{T}>y)$, when $y \rightarrow \infty$, in case $\lambda_{e}<\lambda_{w}^{*}$, i.e., when the information propagation speed is 0 on average.

Lemma 9. When y tends to infinity,

$$
P\left(B_{w}>y\right)=A\left(\lambda_{e}, \lambda_{w}\right) y^{-\frac{\lambda_{e}}{\lambda_{w}}}(1+o(1))
$$

with

$$
A\left(\lambda_{e}, \lambda_{w}\right)=\frac{\lambda_{e} e^{-\lambda_{e}}}{\lambda_{w}^{*} f_{w}\left(\lambda_{e}\right)} \Gamma\left(\frac{\lambda_{e}}{\lambda_{w}^{*}}\right) \beta^{-\frac{\lambda_{e}}{\lambda_{w}^{*}}},
$$

where $\Gamma($.$) is the Euler "Gamma" function, \beta=$ $\frac{\lambda_{w}\left(\lambda_{w}-\lambda_{w}^{*}\right) e^{\lambda_{w}^{*}-2 \lambda_{w}}}{1-\lambda_{w}^{*}}$.

Proof: See appendix.
Lemma 10. When $t \rightarrow \infty$ :

$$
P(\mathbf{T}>t)=A\left(\lambda_{e}, \lambda_{w}\right)(t 2 v)^{-\frac{\lambda_{e}}{\lambda_{w}^{*}}}(1+o(1)) .
$$

Proof: We have the relation $\frac{\mathbf{T}}{2 v}=G_{e w}+B_{w}$, with $G_{e w}=L_{w}^{*}+I_{w}-1$. We know that $G_{e w}$, in analogy with $G_{e}$, has an exponential tail, i.e., $\mathbf{E}\left(e^{-\theta G_{e w}}\right)<\infty$ for all $\theta>-\lambda_{w}$. In other words $P\left(G_{e w}>y\right)=O(\exp (-\theta y))$. The other particularity is that $G_{e w}$ and $B_{w}$ are dependent. First we have the inequality for all $y$

$$
P\left(G_{e w}+B_{w}>y\right) \geq P\left(B_{w}>y\right),
$$

therefore, we have $P(\mathbf{T}>t) \geq A\left(\lambda_{e}, \lambda_{w}\right)(t 2 v)^{-\frac{\lambda_{e}}{\lambda_{w}}}(1+$ $o(1))$. Second, we have the other inequality for all $(y, z)$ :

$$
P\left(G_{e w}+B_{w}>y\right) \leq P\left(G_{e w}>z\right)+P\left(B_{w}>y-z\right) .
$$

Thus, by selecting $z=O(\log t)$ such that $P\left(G_{e w}>z\right)=o\left(t^{-\frac{\lambda_{e}}{\lambda_{w}}}\right)$, we get $P(\mathbf{T}>t) \leq$ $A\left(\lambda_{e}, \lambda_{w}\right)(t-O(\log t) 2 v)^{-\frac{\lambda e}{\lambda_{w}^{*}}}+o\left(t^{-\frac{\lambda_{e}}{\lambda_{w}^{*}}}\right)$

Therefore, we conclude the proof of Theorem 2 .

\section{B. Traveled Distance Distribution}

Now, we focus on the renewal process made of the various waiting time intervals $\mathbf{T}$, experienced by the information beacon. Considering the sequence of waiting phases, $\mathbf{T}_{1}, \mathbf{T}_{2}, \ldots, \mathbf{T}_{n}, \ldots:$ the beacon moves at time $\mathbf{T}_{1}$, then at time $\mathbf{T}_{1}+\mathbf{T}_{2}, \mathbf{T}_{1}+\mathbf{T}_{2}+\mathbf{T}_{3}$, etc. We denote $n(t)$ the number of phases achieved before time $t$ :

$$
\sum_{i=1}^{i=n(t)} \mathbf{T}_{i} \leq t<\sum_{i=1}^{i=n(t)+1} \mathbf{T}_{i} .
$$

Since the $\mathbf{T}_{i}$ are independent, this is a renewal process, and we have the identity:

$$
P(n(t) \leq n)=P\left(\mathbf{T}_{1}+\cdots+\mathbf{T}_{n} \geq t\right) .
$$

We can get a precise estimate of the average number of renewals $\mathbf{E}(n(t))$ during time $t$.

Lemma 11. There exists $b>0$ such that, when $t \rightarrow \infty$, the following estimate is valid:

$$
\mathbf{E}(n(t))=\frac{\sin ^{2}\left(\pi \frac{\lambda_{e}}{\lambda_{w}^{*}}\right)}{b \pi^{2}} \Gamma\left(\frac{\lambda_{e}}{\lambda_{w}^{*}}\right) t^{\frac{\lambda_{e}}{\lambda_{w}}}+O\left(t^{\frac{\lambda_{e}}{\lambda_{w}^{*}}-1}\right) .
$$

Proof: See appendix.

In parallel to the sequence of waiting intervals $\left\{\mathbf{T}_{i}\right\}_{i \geq 1}$, we have the sequence $\left\{\mathbf{D}_{i}\right\}_{i \geq 1}$ the distances traveled by the beacon after every waiting interval $\mathbf{T}_{i}$. Now we denote $L(t)=$ $\sum_{i=1}^{i=n(t)} \mathbf{D}_{i}$ which is the total distance traveled by the beacon until time $t$.

\section{Lemma 12.}

$$
\mathbf{E}(L(t))=\mathbf{E}(n(t)) \mathbf{E}(\mathbf{D}) .
$$

Proof: We have the identity:

$$
\mathbf{E}(L(t))=\sum_{n>0} \mathbf{E}\left(1_{n(t) \geq n} D_{n}\right),
$$


where $1_{n(t) \geq n}$ is the indicative function of the event $n(t) \geq n$. Since, from the definition of $n(t)$, we have the equivalence: $n(t) \geq n \Leftrightarrow \mathbf{T}_{1}+\cdots+\mathbf{T}_{n-1}<t$, then $1_{n \leq n(t)}$ and $D_{n}$ are independent random variables and, therefore, $\mathbf{E}\left(1_{n(t) \geq n} D_{n}\right)=$ $P(n(t) \geq n) \mathbf{E}(\mathbf{D})$.

Quantity $\mathbf{E}(\mathbf{D})$ has a closed expression $\left(\mathbf{E}(\mathbf{D})=\frac{1}{\lambda_{e} f_{w}\left(\lambda_{e}\right)}\right.$, from Corollary 2. Substituting and using Lemma 11, the power law for $\mathbf{E}(L(t))$ in Theorem 3 is shown.

\section{AsYmptotic Estimates}

\section{A. Near the Threshold}

First, we investigate the case where $\left(\lambda_{e}, \lambda_{w}\right)$ is close to the threshold boundary.

Corollary 3. When $\lambda_{e} \rightarrow\left(\lambda_{w}^{*}\right)^{+}$:

$$
v_{p} \sim 2 v \frac{\left(\lambda_{w}-\lambda_{w}^{*}\right) \lambda_{w}}{\lambda_{e}^{2}\left(1-\lambda_{w}^{*}\right) \lambda_{w}^{*}}\left(\lambda_{e}-\lambda_{w}^{*}\right) e^{\lambda_{w}^{*}+\lambda_{e}-2 \lambda_{w}} .
$$

Proof: Using Lemma 6, we have:

$$
\begin{aligned}
2 v E(\mathbf{T})= & -\frac{f_{w}^{\prime}\left(\lambda_{e}\right)}{f_{w}\left(\lambda_{e}\right)}+\int_{1}^{\infty} \mathbf{E}\left(B_{w}(x)\right) p_{e}(x) d x \\
\sim & \int_{1}^{\infty} \frac{e^{\lambda_{w}}}{\lambda_{w}} \frac{\left(1-\lambda_{w}^{*}\right) \lambda_{w}^{*}}{\lambda_{w}-\lambda_{w}^{*}} e^{\lambda_{w}-\lambda_{w}^{*}} e^{(x-1) \lambda_{w}^{*}} \\
& \times \frac{\lambda_{e}}{f_{w}\left(\lambda_{e}\right)} e^{-\lambda_{e} x} d x
\end{aligned}
$$

Integrating, we obtain $v_{p}=\frac{E(\mathbf{D})}{E(\mathbf{T})}$.

\section{B. Large Densities}

Corollary 4. When the vehicle densities become large, i.e., $\lambda_{e}, \lambda_{w} \rightarrow \infty$ :

$$
v_{p} \sim 2 v \frac{e^{\lambda_{e}+\lambda_{w}}}{1+\frac{\lambda_{w}}{\lambda_{e}}+\frac{\lambda_{e}}{\lambda_{w}}}
$$

Proof: According to Lemma 4, we have:

$$
E\left(L_{w}^{*}\right)=1+\frac{\lambda_{w}}{\lambda_{e}\left(\lambda_{w}+\lambda_{e}\right)},
$$

and the expected gap length tends to 1 . The average road length to bridge such a gap tends to $\frac{1}{\lambda_{w}}$. From Lemma 6:

$$
2 v E(\mathbf{T}) \sim E\left(L_{w}^{*}\right)-1+\frac{1}{\lambda_{w}} .
$$

From corollary 2, the average distance traveled in bridging is:

$$
E(\mathbf{D})=\frac{1}{\lambda_{e} f_{w}\left(\lambda_{e}\right)} \sim \frac{e^{\lambda_{e}+\lambda_{w}}}{\lambda_{e}+\lambda_{w}}
$$

and we obtain $v_{p}=\frac{E(\mathbf{D})}{E(\mathbf{T})}$.

Note that the information propagation speed grows quasiexponentially with respect to the total vehicle density.

\section{FINITE RADIO PROPAGATION SPEED}

If the radio propagation speed (including store and forward timings) is finite and constant, equal to $v_{r}$, then the average information propagation speed becomes:

$$
s=\frac{\mathbf{E}\left(T_{w}\right) v+\mathbf{E}\left(D_{e}\right)}{\mathbf{E}\left(T_{w}\right)+\frac{1}{v_{r}} \mathbf{E}\left(D_{e}\right)} .
$$

But the speed $v_{r}$ impacts the random variables $G_{e}$ and $T_{w}$. The main impact is that, to fill an eastbound gap of length $x$, one needs a westbound cluster of length at least $\frac{x(1+\gamma)}{1-\gamma}$ with $\gamma=$ $\frac{v}{v_{r}}$, otherwise the message will be off the gap when arriving at the end of the cluster. Thus, $\mathbf{E}\left(T_{w}(x)\right)=O\left(e^{\frac{(1+\gamma) \theta_{w}}{1-\gamma} x}\right)$.

The gap length is also modified, since we must consider $\frac{1-\gamma}{1+\gamma} L_{w}^{*}$ :

$$
\mathbf{E}\left(e^{-\theta G_{e}}\right)=\frac{f_{w}\left(\frac{1-\gamma}{1+\gamma}\left(\theta+\lambda_{e}\right)\right)}{f_{w}\left(\frac{1-\gamma}{1+\gamma} \lambda_{e}\right)} \frac{\lambda_{e}}{\lambda_{e}+\theta} .
$$

But, this does not change the exponential term $e^{-\lambda_{e} x}$ in the asymptotic expression of $p_{e}(x)$. Therefore, the threshold condition becomes:

$$
\lambda_{w} e^{-\lambda_{w}}=\frac{1-\gamma}{1+\gamma} \lambda_{e} e^{-\frac{1-\gamma}{1+\gamma} \lambda_{e}} .
$$

This corresponds to a dilatation by a factor $\frac{1+\gamma}{1-\gamma}$ of the horizontal axis in the diagram of Figure 2. Notice how the diagram then loses its symmetry with respect to $\lambda_{e}$ versus $\lambda_{w}$, which can be observed in Figure 9.

Notice also that, when $v_{r}$ decreases to $v$, the threshold limit tends to infinity.

Similarly, in correspondance to Theorem 3, we have $\mathbf{E}(L(t))=\Omega\left(t^{\frac{1-\gamma}{1+\gamma} \frac{\lambda_{e}}{\lambda_{w}^{*}}}\right)$, due to the dilatation by the factor $\frac{1+\gamma}{1-\gamma}$.

\section{Simulations}

In this section, we present simulation results obtained with Maple on one hand, and the Opportunistic Network Environment (ONE [14]) simulator on the other hand.

\section{A. Maple Simulations}

We first compare the theoretical analysis with measurements performed using Maple. In this case, the simulations follow precisely the bidirectional highway model described in Section II: we generate Poisson traffic of eastbound and westbound traffic on two opposite lanes moving at constant speed, which is set to $v=1 \mathrm{~m} / \mathrm{s}$. The radio propagation range is $R=1 \mathrm{~m}$, and radio transmissions are instantaneous; the length of the highway is sufficiently large to provide a large number of bridging operations (of order at least $10^{3}$ ) for all considered traffic densities.

We measure the information propagation speed which is achieved using optimal DTN routing, by selecting a source and destination pairs at large distances, taking the ratio of the propagation distance over the corresponding delay, and averaging over multiple iterations of randomly generated traffic. We vary the total traffic density, and we plot the resulting 


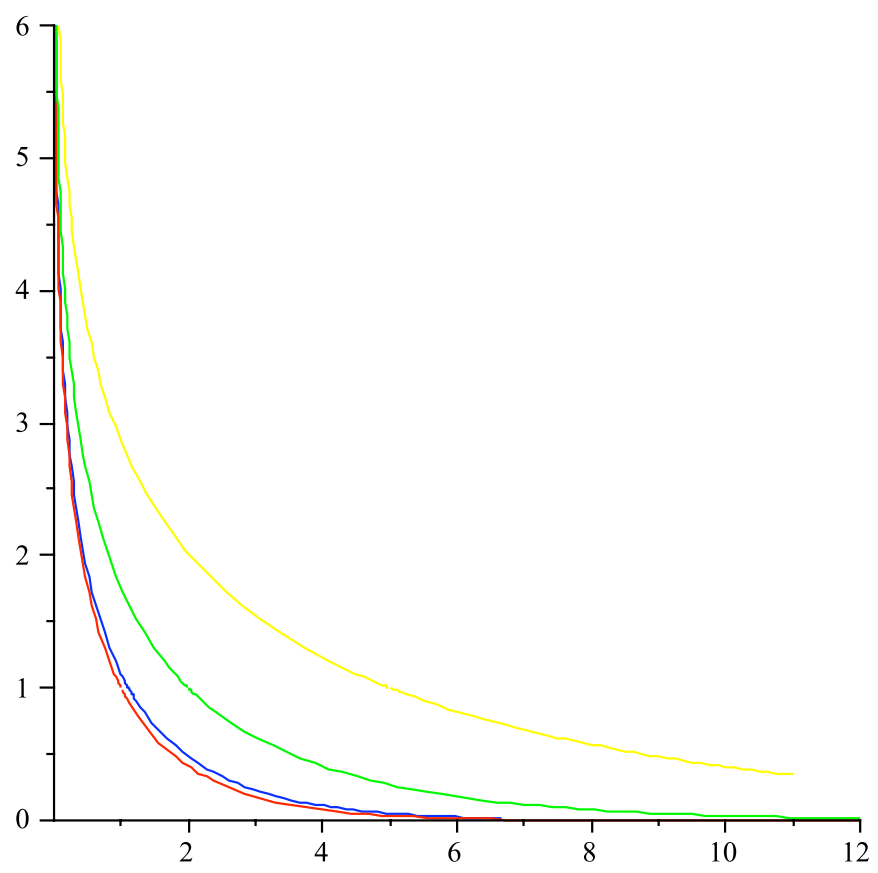

Fig. 9. Threshold zones for $\left(\lambda_{e}, \lambda_{w}\right)$ for various $\gamma=\frac{v}{v_{r}}, \gamma=0$ red, $\gamma=0.1$ blue, $\gamma=0.5$ green, $\gamma=0.8$ yellow.

information propagation speed. Figures 10 and 11 show the evolution of the information propagation speed near the threshold versus the total vehicle density, when $\lambda_{e}=\lambda_{w}$, in linear and semilogarithmic plots, respectively. We can observe the threshold at $\lambda_{e}+\lambda_{w}=2$ in Figure 10, which confirms the analysis presented previously in Section III, and corresponds to $\lambda_{e}=\lambda_{w}=1$ in Figure 2. In semilogarithmic scale (Figure 11), we observe that the simulation measurements quickly approach a straight line, and therefore are close to the theoretically predicted exponential growth above the phase transition threshold, in Section V. In Figure 12, we present a 3 -dimensional plot of the eastbound information propagation speed $v_{p}$ by varying the vehicle densities in both eastbound $\left(\lambda_{e}\right)$ and westbound $\left(\lambda_{w}\right)$ traffic.

Finally, we perform detailed measurements of the waiting time $\mathbf{T}$ that each packet of information spends in the buffer of an eastbound car until it encounters a westbound cluster which allows it to propagate faster to the next eastbound car. For the measurements, we set the vehicle densities to $\lambda_{e}=\lambda_{w}=0.9$; thus the conjugate $\lambda_{w}^{*}=1.107 \ldots$ In Figure 13, (i.e., below the phase transition threshold), we plot the distribution of the waiting time $\mathbf{T}$, and we compare it to the predicted power law in Theorem 2: $P(\mathbf{T}>t)=t^{-\frac{\lambda_{e}}{\lambda_{w}^{*}}}$.

\section{B. ONE Simulations}

In this section, we depart from the exact Poisson model simulations in Maple, and we present simulation results obtained with the Opportunistic Network Environment (ONE [14]) simulator. Vehicles are distributed uniformly on the length of both lanes of a road, and move at a constant unit speed. The total

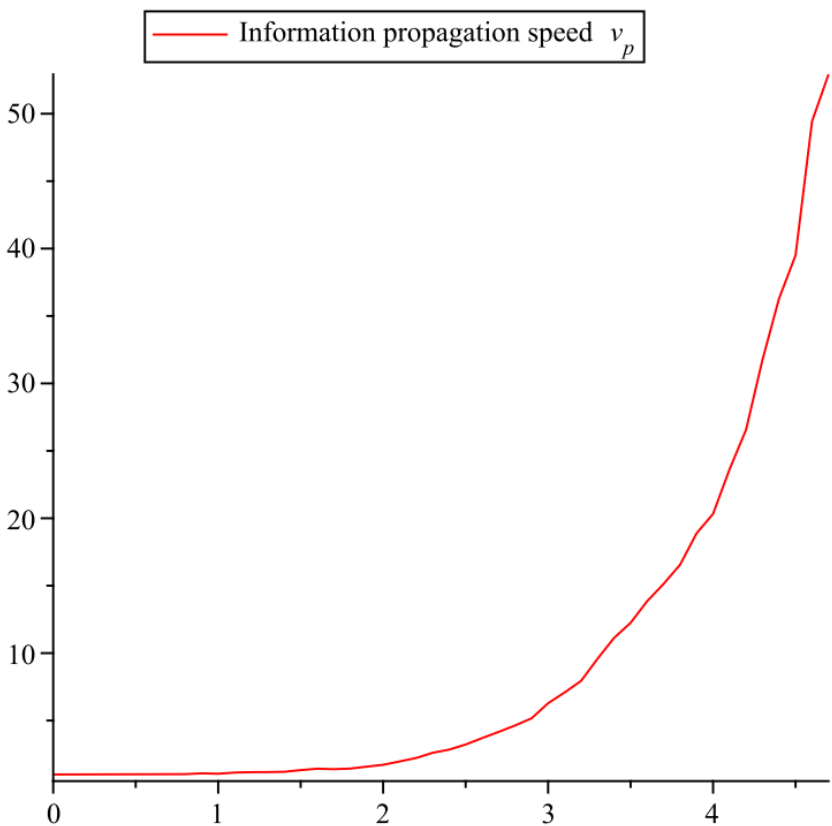

Fig. 10. Maple simulations. Information propagation speed $v_{p}$ for $\lambda_{e}=\lambda_{w}$, versus the total vehicle density $\lambda_{e}+\lambda_{w}$.

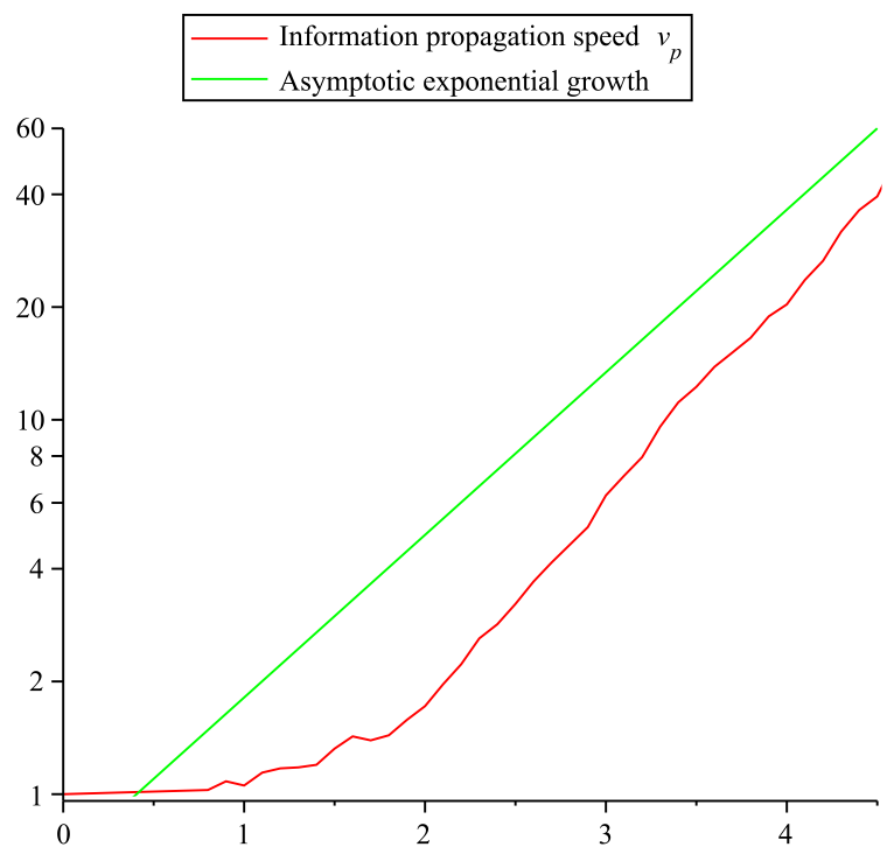

Fig. 11. Maple simulations. Information propagation speed $v_{p}$ for $\lambda_{e}=\lambda_{w}$, versus the total vehicle density $\lambda_{e}+\lambda_{w}$, in semi-log scale, compared to the theoretically predicted asymptotic exponential growth. 


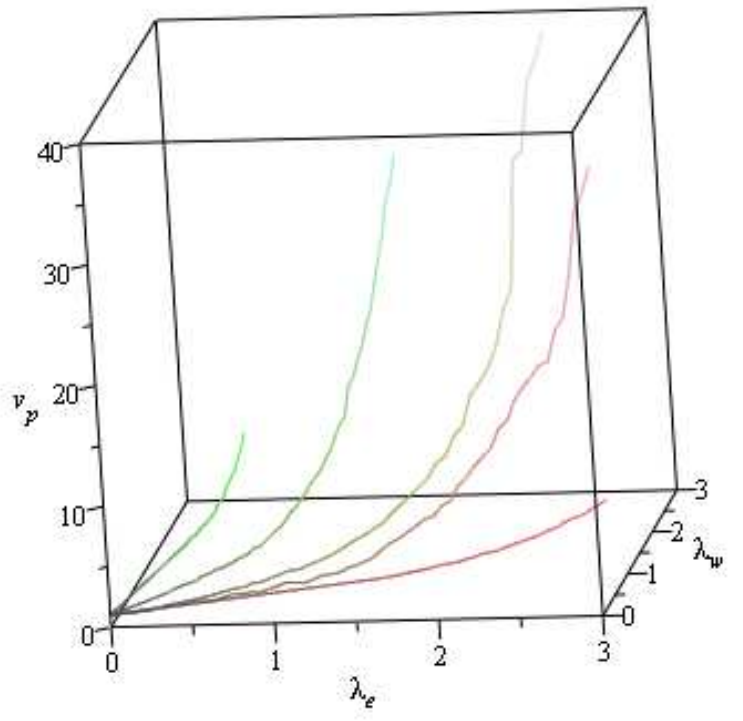

Fig. 12. Maple simulations. Eastbound information propagation speed $v_{p}$ for different values of the vehicule densities, $\lambda_{e}$ and $\lambda_{w}$.
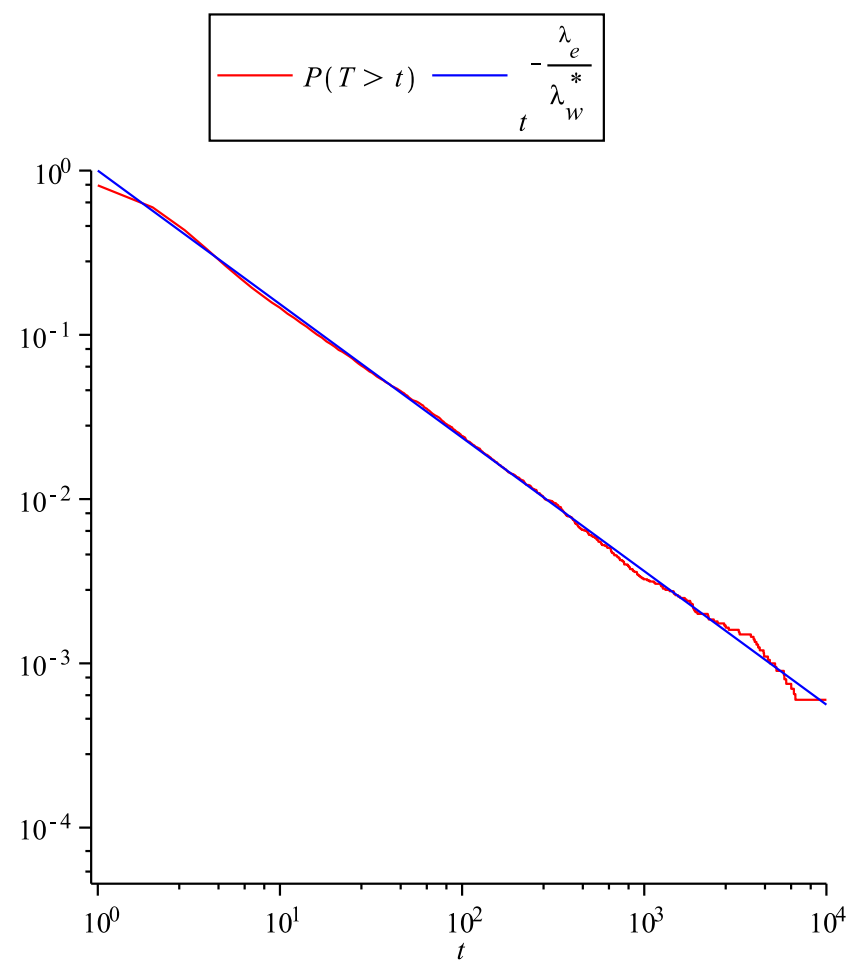

Fig. 13. Maple simulations. Cumulative probability distribution $P(\mathbf{T}>t)$ of the waiting time $\mathbf{T}$, compared to the power law $t^{-\frac{\lambda_{e}}{\lambda_{w}^{*}}}$, for $\lambda_{e}=\lambda_{w}=0.9$.

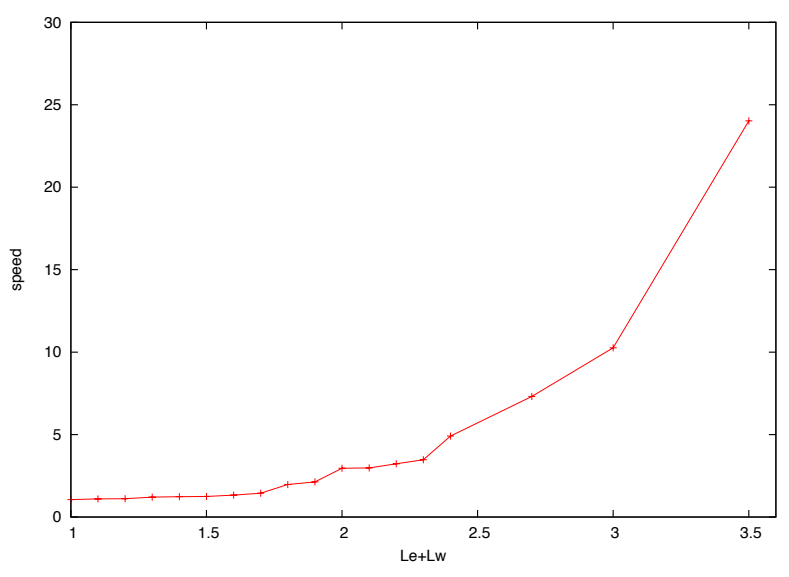

Fig. 14. ONE simulations. Information propagation speed for $\lambda_{e}=\lambda_{w}$, with respect to $\lambda_{e}+\lambda_{w}$.

number of vehicles varies from 1000 to 5000 . Similarly to the previous section, we measure the fastest possible information propagation speed which is achieved using epidemic broadcast, assuming that radio transmissions are instantaneous and that there are no buffering or congestion delays, with a radio range $R=10 \mathrm{~m}$. Again, we vary the vehicle densities $\lambda_{e}$ and $\lambda_{w}$, which are given in vehicles per radio range, and we perform several simulation iterations of randomly generated traffic. As shown in Figure 14, similarly to the Maple simulation results and to the analysis, we observe the threshold phenomenon at $\lambda_{e}=\lambda_{w}=1$ : the information propagation speed remains almost constant below the threshold but increases dramatically beyond it. We also observe an exponential growth above the threshold in Figure 15.

We remark that measurements below the phase transition threshold yield an average information propagation speed which is slightly larger than the vehicle speed. This is due to the finite duration of the simulations and in the computations of the expectations. This phenomenon can also be explained from the theoretical analysis in Section IV: even below the threshold, DTN routing using cars moving on both directions still provides a gain in the propagation distance, which follows a sub-linear power law with respect to the elapsed time (in the referential of the moving cars). Figure 16 confirms the predicted power law $t^{\lambda_{e} / \lambda_{w}^{*}}$ for $\mathbf{E}(L(t))$, the average distance traveled by information with respect to time (in Theorem 3), which is shown in Figure 16 for $\lambda_{e}=1$ and $\lambda_{w}=0.9$, therefore, a growth of order $t^{0.903}$.

\section{CONCLUdING REMARKS}

In this paper, we provided a detailed analysis for information propagation in bidirectional vehicular DTNs. We proved the existence of a threshold, concerning vehicle density, above which information speed increases dramatically over vehicle speed, and below which information propagation speed 


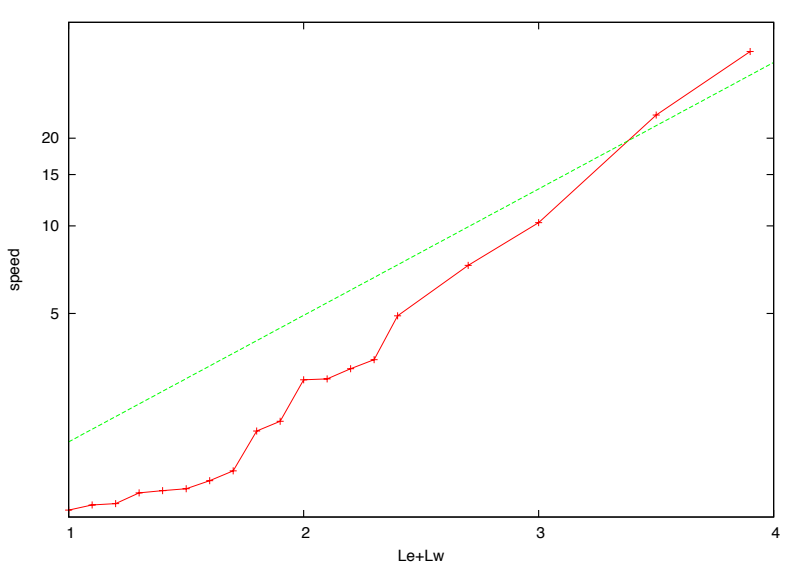

Fig. 15. ONE simulations. Information propagation speed for $\lambda_{e}=\lambda_{w}$, with respect to $\lambda_{e}+\lambda_{w}$, in semi-log scale, compared to the theoretically predicted asymptotic exponential growth.

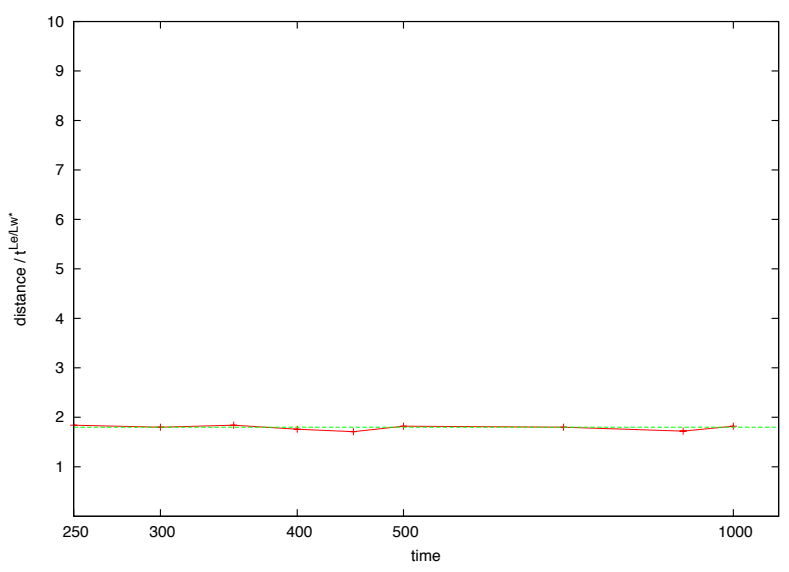

Fig. 16. ONE simulations. Average distance traveled by information with respect to elapsed time $\mathbf{E}(L(t))$, divided by the predicted power law $t^{\lambda_{e} / \lambda_{w}^{*}}$ for $\lambda_{e}=1$ and $\lambda_{w}=0.9$, compared to the constant value 1.80 (dash).

is on average equal to vehicle speed (in Theorem 1), and we computed the exact expression of this threshold. We exactly characterized the information speed near the threshold (Corollary 3), and we showed that, above the threshold, the information propagation speed increases quasi-exponentially with vehicle density (Corollary 4). We also analyzed in detail the way information propagates under the threshold, and we showed that DTN routing using bidirectional traffic provides a gain in the propagation distance, which follows a sub-linear power law with respect to the elapsed time (Theorems 2 and 3). Combining all these different situations, we obtain a complete image of the way information propagates in vehicular networks on roads and highways, which is useful in determining the per- formance limits and designing appropriate routing protocols for VANETs. All our theoretical results were validated with simulations in several environments (The One and Maple).

Our analysis can be extended to investigate other models of vehicle traffic and radio propagation. In future works, we intend to provide a detailed expression of this threshold in specific VANET models (e.g., intersections). Finally, an interesting direction for further research consists in collecting large traces of real traffic on roads and highways, and evaluating the information propagation properties in this context.

\section{REFERENCES}

[1] A. Agarwal, D. Starobinski and T. Little, "Phase Transition Behavior of Message Propagation in Delay Tolerant Vehicular Ad Hoc Networks". MCL Technical Report No. 12-12-2008, 2008.

[2] A. Agarwal, D. Starobinski and T. Little, "Analytical Model for Message Propagation in Delay Tolerant Vehicular Networks". Proceedings of the Vehicular Technology Conference (VTC). Singapore, 2008.

[3] A. Agarwal, D. Starobinski and T. Little, "Exploiting Mobility to Achieve Fast Upstream Propagation". Proceedings of Mobile Networking for Vehicular Environments (MOVE) at IEEE INFOCOM. Anchorage, 2007.

[4] E. Baccelli, P. Jacquet, B. Mans and G. Rodolakis, "Information Propagation Speed in Bidirectional Vehicular Delay Tolerant Networks", Infocom, 2011.

[5] J. Burgess, B. Gallagher, D. Jensen and B. Levine, "MaxProp: Routing for Vehicle-Based Disruption-Tolerant Networks". In Infocom, 2006.

[6] A. Casteigts, A. Nayak and I. Stojmenovic, "Communication protocols for vehicular ad hoc networks". Wireless Communications and Mobile Computing,

[7] V. Cerf, S. Burleigh, A. Hooke, L. Torgerson, R. Durst, K. Scott, K. Fall and H. Weiss, "Delay-Tolerant Networking Architecture", IETF Request For Comment RFC 4838, Internet Engineering Task Force, 2007.

[8] F. De Pellegrini, D. Miorandi, I. Carreras and I. Chlamtac, "A Graphbased model for disconnected ad hoc networks", Infocom, 2007.

[9] P. Flajolet, A. Odlyzko, "Singularity analysis of generating functions", SIAM Journal Disc. Math.,Vol. 3, No. 2, pp. 216-240, May 1990.

[10] R. Fracchia and M. Meo, "Analysis and Design of Warning Delivery Service in Inter-vehicular Networks". IEEE Transactions on Mobile Computing. 2008.

[11] M. Grossglauser and D. Tse, "Mobility increases the capacity of ad hoc wireless networks", Infocom, 2001.

[12] P. Gupta and P. R. Kumar, "The capacity of wireless networks", IEEE Trans. on Info. Theory, vol. IT-46(2), pp. 388-404, 2000.

[13] P. Jacquet, B. Mans and G. Rodolakis, "Information propagation speed in mobile and delay tolerant networks", Infocom, 2009.

[14] A. Keranen, J. Ott and T. Karkkainen, "The ONE Simulator for DTN Protocol Evaluation". SIMUTools'09: 2nd International Conference on Simulation Tools and Techniques. Rome, 2009.

[15] Z. Kong and E. Yeh, "On the latency for information dissemination in Mobile Wireless Networks", MobiHoc, 2008.

[16] T. Nadeem, P. Shankar, and L. Iftode, "A Comparative Study of Data Dissemination Models for VANETs", in Proc. of MOBIQUITOUS, 2006.

[17] Y. Toor, P. Mühlethaler, A. Laouiti, A. de la Fortelle, "Vehicle ad hoc networks: Applications and related technical issues". IEEE Communications Surveys and Tutorials 10(1-4), pp. 74-88 (2008)

[18] US-Department of Transportation. National Transportation Statistics, 2010. US Government printing Office, Washington, DC.

[19] N. Wisitpongphan, F. Bai, P. Mudalige, and O. Tonguz, "On the Routing Problem in Disconnected Vehicular Ad-hoc Networks," in Infocom, 2007.

[20] H. Wu, R. Fujimoto, and G. Riley, "Analytical Models for Information Propagation in Vehicle-to-Vehicle Networks", in Proc. Fall VTC 04, Los Angeles, CA, USA, 2004.

[21] S. Yousefi, E. Altman, R. El-Azouzi and M. Fathy, "Analytical Model for Connectivity in Vehicular Ad Hoc Networks". IEEE Transactions on Vehicular Technology. Nov. 2008.

[22] X. Zhang, G. Neglia, J. Kurose and D. Towsley, "Performance modeling of epidemic routing", Computer Networks, Vol. 51, 2007. 


\section{APPENDIX}

\section{A. Proof of Lemma 9}

Proof: We want to find an evaluation of $P(\mathbf{T}>y)$ when $y \rightarrow \infty$. For this we will evaluate for $x$ given $P\left(B_{w}(x)>y\right)$. We know that

$$
\mathbf{E}\left(e^{\theta B_{w}(x+1)}\right)=\beta_{w}(\theta, x+1)=\frac{\lambda_{w} P\left(L_{w}>x\right)}{\theta-\lambda_{w}\left(f_{w}(\theta, x+1)-1\right)}
$$

Since $P\left(L_{w}>x\right)=\frac{\alpha}{\lambda_{w}^{*}} e^{-\lambda_{w}^{*} x}\left(1+o\left(e^{-\varepsilon x}\right)\right)$, with $\alpha=$ $\frac{\left(\lambda_{w}-\lambda_{w}^{*}\right) e^{\lambda_{w}^{*}-\lambda_{w}}}{1-\lambda_{w}^{*}}$, we have

$$
f_{w}(\theta, x)=f_{w}(\theta)-\frac{\alpha e^{-\lambda_{w}^{*} x}}{\theta+\lambda_{w}^{*}}\left(1+o\left(e^{-\varepsilon x}\right)\right)
$$

From here we drop the $o\left(e^{-\varepsilon x}\right)$ for simplicity, since it will just bring an exponentially small factor. Therefore we have

$$
\beta_{w}(\theta, x+1)=\frac{\lambda_{w}}{\lambda_{w}^{*}} \frac{\alpha e^{-\lambda_{w}^{*} x}}{\theta-\lambda_{w}\left(f_{w}(\theta)-1-\frac{\alpha e^{-\lambda_{w}^{*} x}}{\theta+\lambda_{w}^{*}}\right)}
$$

We have

$$
P\left(B_{w}(x+1)=y\right)=\frac{1}{2 i \pi} \int \beta_{w}(\theta, x+1) e^{y \theta} d \theta
$$

Let $\theta(x)$ be the root of $\theta-\lambda_{w}\left(f_{w}(\theta)-1-\frac{\alpha e^{-\lambda_{w}^{*} x}}{\theta+\lambda_{w}^{*}}\right)$. Straightforward analysis gives $\theta(x)=-\beta e^{-\lambda_{w}^{*} x}+O\left(e^{-2 \lambda_{w}^{w} x}\right)$, with $\beta=\frac{\lambda_{w}}{1-\lambda_{w} \frac{\partial}{\partial \theta} f_{w}(0)} \frac{\alpha}{\lambda_{w}^{*}}$. Via singularity analysis we have

$$
\begin{aligned}
P\left(B_{w}(x+1)=y\right)= & \frac{\lambda_{w} \alpha e^{-\lambda_{w}^{*} x}}{\lambda_{w}^{*}} \\
& \times \frac{e^{\theta(x) y}}{1-\lambda_{w} \frac{\partial}{\partial \theta} f_{w}(\theta(x))-\frac{\alpha e^{-\lambda_{w}^{*} x}}{\left(\theta(x)+\lambda_{w}^{*}\right)^{2}}} \\
& +O\left(e^{(\theta(x)-\varepsilon) y}\right)
\end{aligned}
$$

Omitting the $O()$ terms we get

$$
\begin{aligned}
P\left(B_{w}(x+1)=y\right) & =\frac{\lambda_{w} \alpha e^{-\lambda_{w}^{*} x}}{\lambda_{w}^{*}} \frac{e^{-\beta e^{-\lambda_{w}^{*} x} y}}{1-\lambda_{w} \frac{\partial}{\partial \theta} f_{w}(0)} \\
& =\beta e^{-\theta x} \exp \left(-\beta e^{-\lambda_{w}^{*} x} y\right)
\end{aligned}
$$

Or

$$
P\left(B_{w}(x+1)>y\right)=\exp \left(-\beta e^{-\lambda_{w}^{*} x} y\right)
$$

Therefore stating $P\left(B_{w}>y\right)=\int p_{e}(x) P\left(B_{w}(x)>y\right) d x$ we get, omitting $O($ ) terms

$$
P\left(B_{w}>y\right)=\int \frac{\lambda_{e}}{f_{w}\left(\lambda_{e}\right)} e^{-(x+1) \lambda_{e}} \exp \left(-\beta e^{-\lambda_{w}^{*} x} y\right) d x .
$$

with the change of variable $u=e^{-\lambda_{w}^{*} x}$ we get

$$
\begin{aligned}
P\left(B_{w}>y\right) & =\int \frac{\lambda_{e} e^{-\lambda_{e}}}{\lambda_{w}^{*} f_{w}\left(\lambda_{e}\right)} u^{\frac{\lambda_{e}}{\lambda_{w}^{*}}-1} \exp (-\beta y u) d u \\
& =\frac{\lambda_{e} e^{-\lambda_{e}}}{\lambda_{w}^{*} f_{w}\left(\lambda_{e}\right)} \Gamma\left(\frac{\lambda_{e}}{\lambda_{w}^{*}}\right)(\beta y)^{-\frac{\lambda_{e}}{\lambda_{w}^{*}}}
\end{aligned}
$$

which is in power law as claimed.

\section{B. Proof of Lemma 11}

We first prove the property for an hypothetic renewal process based on the $B_{i}^{\prime} s$ on the real line. Let $b(x)$ be this renewal process at time $t$ :

$$
\begin{aligned}
\mathbf{E}(b(y))= & P\left(B_{1}<y\right)+P\left(B_{1}+B_{2}<y\right) \\
& +\cdots+P\left(B_{1}+\cdots+B_{n}<y\right)+\cdots
\end{aligned}
$$

Let us define $\beta=1-\mathbf{E}\left(e^{-\theta B_{w}}\right)$. We also define $N_{B}(\theta)=$ $\int_{0}^{\infty} \mathbf{E}(b(y)) e^{-y \theta}$ the Laplace transform of $\mathbf{E}(b(y))$. We have:

$$
N_{B}(\theta)=\frac{1-B(\theta)}{\theta B(\theta)} .
$$

Lemma 13. When $\theta \rightarrow 0$, then $B(\theta)=b \frac{\pi \theta^{a}}{\sin (\pi a)}$ for some $b$ and $a=\frac{\lambda_{e}}{\lambda_{w}^{*}}$.

Proof: We start from

$$
\beta(\theta, x+1)=\frac{\lambda_{w} P\left(L_{w}>x+1\right)}{\theta-\lambda_{w}\left(f_{w}(\theta, x+1)-1\right)},
$$

and

$B(\theta)=1-\int_{1}^{\infty} p_{e}(x) \beta(\theta, x) d x=\int_{1}^{\infty} p_{e}(x)(1-\beta(\theta, x)) d x$.

We denote

$$
1-\beta(\theta, x)=\frac{\theta}{\theta+\theta(x)} g(\theta, x)
$$

with $g(\theta, x)$ bounded and uniformly integrable when $\Re(\theta)$ remains in a compact set and $x \rightarrow \infty$. Indeed we have

$$
g(\theta, x)=1+O(\theta(x))
$$

Thus

$$
B(\theta)=\int_{1}^{\infty} \frac{\theta}{\theta+\theta(x)} g(\theta, x) p_{e}(x) .
$$

By change of variable $y=\theta(x)$ we get

$$
B(\theta)=\int_{0}^{\theta(1)} \frac{\theta}{\theta+y} g\left(\theta, \theta^{-1}(y)\right) p_{e}\left(\theta^{-1}(y)\right)\left(\theta^{-1}(y)\right)^{\prime} d y .
$$

We use together the following estimates $\theta(x)=-\beta e^{-\lambda_{w}^{*} x}+$ $O\left(e^{-2 \lambda_{w}^{*} x}\right)$, and $p_{e}(x)=\frac{\lambda_{e}}{f_{w}\left(\lambda_{e}\right)} e^{-\lambda_{e} x}\left(1+O\left(e^{-\varepsilon x}\right)\right)$ to state

$$
p_{e}\left(\theta^{-1}(y)\right)\left(\theta^{-1}(y)\right)^{\prime}=\frac{\lambda_{e}}{f_{w}\left(\lambda_{e}\right)}\left(\frac{y}{\beta}\right)^{\frac{\lambda_{e}}{\lambda_{w}^{w}}-1}\left(1+O\left(y^{\varepsilon}\right)\right),
$$

for some $\varepsilon>0$.

Then, we use the fact that

$$
\int_{0}^{\infty} \frac{y^{a-1}}{\theta+y} d y=\frac{\pi \theta^{a-1}}{\sin (\pi a)}
$$

and, when $\theta \rightarrow 0$,

$$
\int_{\theta(1)}^{\infty} \frac{y^{a-1}}{\theta+y} d y=O(1)
$$

Therefore, $B(\theta)=b \frac{\pi \theta^{a}}{\sin (\pi a)}+O(\theta)$ for some $b$ and $a=\frac{\lambda_{e}}{\lambda_{w}^{*}}$ when $\theta \rightarrow 0$. This is also true for complex $\theta$.

We can now prove Lemma 11 for the original renewal process. 
Proof: From the previous lemma, we have that: $N_{B}(\theta)=$ $\frac{\sin (\pi a)}{b \pi} \theta^{-a-1}+O\left(\theta^{-1}+\theta^{-2 a}\right)$, when $\theta \rightarrow 0$.

The inversion of the Laplace transform yields

$$
\mathbf{E}(b(y))=\frac{1}{2 i \pi} \int_{c-i \infty}^{c+i \infty} N_{B}(\theta) e^{\theta y} d \theta .
$$

for any $c>0$. we bend the integration path so that it resemble the path described in figure 17 with $c^{\prime}<0$. Since far from its singularities on the line $-\theta(x)$ for $x>0$ (which corresponds to the negative real axis), the functions $\beta(\theta, x)$ is uniformly in $\frac{1}{\theta}$, therefore $N_{B}(\theta)=O\left(\frac{1}{\theta^{2}}\right)$ and the integral on the vertical part gives a bounded contribution (in fact exponentially decreasing in $\exp \left(c^{\prime} y\right)$ ).

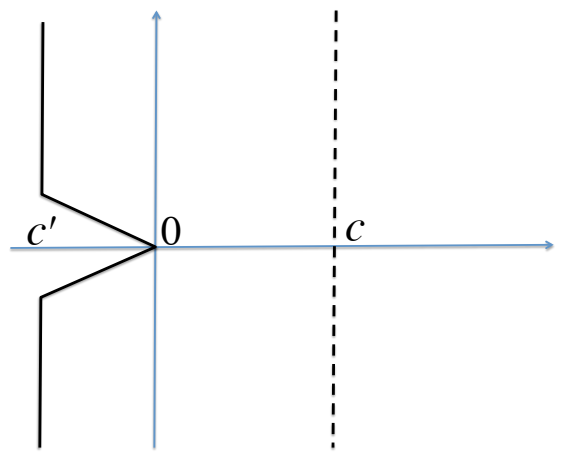

Fig. 17. The Flajolet-Odlyzko cranted integral path.

The cranted part of the integral, using the Flajolet Odlyzko theorem on continuous functions [9], gives a contribution which is

$$
\frac{\sin ^{2}(\pi a)}{b \pi^{2}} \Gamma(-a) t^{a}+O\left(1+t^{2 a-1}\right)
$$

Therefore, we obtain that, for $y \rightarrow \infty$ :

$$
\mathbf{E}(b(y))=\frac{\sin ^{2}(\pi a)}{b \pi^{2}} \Gamma(-a) y^{a}+O\left(1+y^{2 a-1}\right) .
$$

To terminate the argument we will prove that $\mathbf{E}(n(t))=$ $\mathbf{E}(b(2 v t))+o(1)$. We have $2 v \mathbf{T}=B_{w}+H_{w}$, where $H_{w}=$ $L_{w}+I_{w}-1$, and $H_{w} \geq 0$ has distribution with an exponentially decreasing tail. However, we stress the fact that $B_{w}$ and $H_{w}$ are not independent. We denote $H_{i}$ the successive values of the $H_{w}$. We have therefore $2 v \mathbf{T}_{i}=B_{i}+H_{i}$.

Since $\mathbf{T}_{i} \geq 2 v B_{i}$, we already have $\mathbf{E}(n(t)) \leq \mathbf{E}(b(2 v t))$. We also have for any $t^{\prime} \in[0, t]$ and for any integer $i$ :

$$
\begin{aligned}
P\left(\mathbf{T}_{1}+\cdots+\mathbf{T}_{i}<t\right) \geq & P\left(B_{1}+\cdots+B_{i}<2 v\left(t-t^{\prime}\right)\right) \\
& -P\left(H_{1}+\cdots+H_{i}>2 v t^{\prime}\right) .
\end{aligned}
$$

Using Chernoff bounds, we have for all $\theta>0$ such that $\mathbf{E}\left(e^{\theta H}\right)$ exists, $\forall i \leq k$ and $\forall x>0$ :

$$
P\left(H_{1}+\cdots+H_{i}>x\right) \leq \mathbf{E}(\exp (k \theta H-x \theta))
$$

Since $\log \mathbf{E}\left(e^{\theta H}\right)=\theta \mathbf{E}(H)+O\left(\theta^{2}\right)$, it is sufficient that $\frac{x}{k}-$ $\mathbf{E}(H)>\gamma$, to find $\theta>0$ such that $\log P\left(H_{1}+\cdots+H_{i}>\right.$ $x)<-k \gamma \theta$.

On the other side, we also have $\forall y>0$ :

$\sum_{i \leq k} P\left(B_{1}+\cdots+B_{i}<y\right)=\mathbf{E}(b(y))-\sum_{i>k} P\left(B_{1}+\cdots+B_{i}<y\right)$

We have

$$
P\left(B_{1}+\cdots+B_{i}<y\right) \leq(P(B<y))^{i}
$$

We use the fact that $P(B>y) \leq A y^{-a}$ for $a=\frac{\lambda_{e}}{\lambda_{w}^{*}}$ and for some $A>0$ to state that

$$
P\left(B_{1}+\cdots+B_{i}<y\right) \leq \exp \left(-i A y^{-a}\right)
$$

and, finally,

$$
\sum_{i>k} P\left(B_{1}+\cdots+B_{i}<y\right) \frac{\exp \left(-k A y^{-a}\right)}{A y^{-a}} .
$$

Collecting all results yields:

$$
\mathbf{E}(n(t)) \geq \mathbf{E}\left(b\left(2 v\left(t-t^{\prime}\right)\right)\right)-G_{k}\left(2 v t, 2 v t^{\prime}, \theta\right),
$$

with

$$
G_{k}(x, y, \theta)=\frac{\exp \left(-k A(y-x)^{-a}\right)}{A(y-x)^{-a}}+k \mathbf{E}(\exp (k \theta H-x \theta)) .
$$

If we take $t^{\prime}=t^{a^{\prime}}$ with $1>a^{\prime}>a, k=\frac{2 v t^{\prime}}{\gamma+\mathbf{E}(H)}=\Omega\left(t^{a^{\prime}}\right.$, then we have

$$
G\left({ }_{k}\left(2 v t^{\prime}, 2 v t, \theta\right) \leq \frac{\exp \left(-k\left(2 v\left(t-t^{\prime}\right)\right)^{-a}\right)}{A\left(2 v\left(t-t^{\prime}\right)\right)^{-\alpha}}+k \exp (-k \gamma \theta)\right.
$$

which is exponentially small when $t \rightarrow \infty$. Therefore,

$$
\mathbf{E}(n(t)) \geq \mathbf{E}(b(t))+o(1) .
$$

